\title{
sciendo
}

\section{Application of genetic algorithms in optimization of SFR nuclear reactor design}

Wojciech Żurkowski, Piotr Sawicki,

Wojciech Kubiński (1), Piotr Darnowski

\begin{abstract}
This work presents a demonstrational application of genetic algorithms (GAs) to solve sample optimization problems in the generation IV nuclear reactor core design. The new software was developed implementing novel GAs, and it was applied to show their capabilities by presenting an example solution of two selected problems to check whether GAs can be used successfully in reactor engineering as an optimization tool. The 3600 MWth oxide core, which was based on the OECD/NEA sodium-cooled fast reactor (SFR) benchmark, was used a reference design [1]. The first problem was the optimization of the fuel isotopic inventory in terms of minimizing the volume share of long-lived actinides, while maximizing the effective neutron multiplication factor. The second task was the optimization of the boron shield distribution around the reactor core to minimize the sodium void reactivity effect (SVRE). Neutron transport and fuel depletion simulations were performed using Monte Carlo neutron transport code SERPENT2. The simulation resulted in an optimized fuel mixture composition for the selected parameters, which demonstrates the functionality of the algorithm. The results show the efficiency and universality of GAs in multidimensional optimization problems in nuclear engineering.
\end{abstract}

Keywords: Genetic algorithms $\bullet$ Nuclear reactor $\bullet$ Optimization $\bullet$ Sodium-cooled fast reactor

W. Żurkowski ${ }^{\bowtie}$, P. Sawicki

Warsaw University of Technology

Faculty of Physics

Koszykowa 75 Str., 00-665, Warsaw, Poland

E-mail: zurkowski@outlook.com

W. Kubiński

National Centre for Nuclear Research

Andrzeja Sołtana 7 Str., 05-400 Otwock-Świerk, Poland

P. Darnowski

Warsaw University of Technology

Institute of Heat Engineering

Nowowiejska 21/25 Str., 00-665, Warsaw, Poland

Received: 19 January 2021

Accepted: 21 April 2021

\section{Introduction}

The fourth generation of nuclear reactors is the next step in nuclear power development, where the most important goal is to increase fissile resource utilization, sustainability, and improve safety [2-6]. The sodium-cooled fast reactor (SFR) technology, studied in this work, gained the largest operating experience [7-10].

Genetic algorithms (GAs) [11, 12] are exploratory tools that draw inspiration from genetics and theory of evolution. GAs implement natural selection mechanisms and the principle of heredity to solve complex optimization problems. They are especially useful in the design process, as they are universal, simple to implement, and global, unlike other popular methods; therefore, GAs were used in several studies related to nuclear engineering [13-18].

This study is focused on the sample application of the developed GAs to solve two examples of core design-related problems. Problems were defined to demonstrate the abilities of the developed GAs and test their implementations.

0029-5922 @ 2021 The Author(s). Published by the Institute of Nuclear Chemistry and Technology.

This is an open access article under the CC BY-NC-ND 4.0 licence (http://creativecommons.org/licences/by-nc-nd/4.0/). 


\section{Methodology}

The basic approach of GAs is based on three simple principles of natural selection. Each population that is created is subjected to an environmental adaptation assessment. Similar to nature, GAs are based on data arrays, by analogy called chromosomes. Commonly, they are represented as a string using a vector of $n$ binary bits. Chromosomes contain information about the full genotype (system). Next, these arrays can be selected to form offspring via the crossover process, which involves exchanging genes of two selected chromosomes. The higher the fitness of each individual, the higher are the chances of being selected. Offspring can additionally be mutated, altering their genes with low probability (usually $<1 \%$ per gene). Then, depending on the value of fitness function (FF), new chromosomes may or may not be selected for crossover [12]. As a consequence of taking only the strongest chromosomes, only they are able to create offspring. The result of combining this operation with the possibility of mutation is the development of a new generation and - after a certain number of cycles - an optimal solution. The general scheme of the GA implementation is presented in Fig. 1.

\section{Fitness function}

It is a fundamental tool that assesses each individual from the population. Its formula depends strictly on

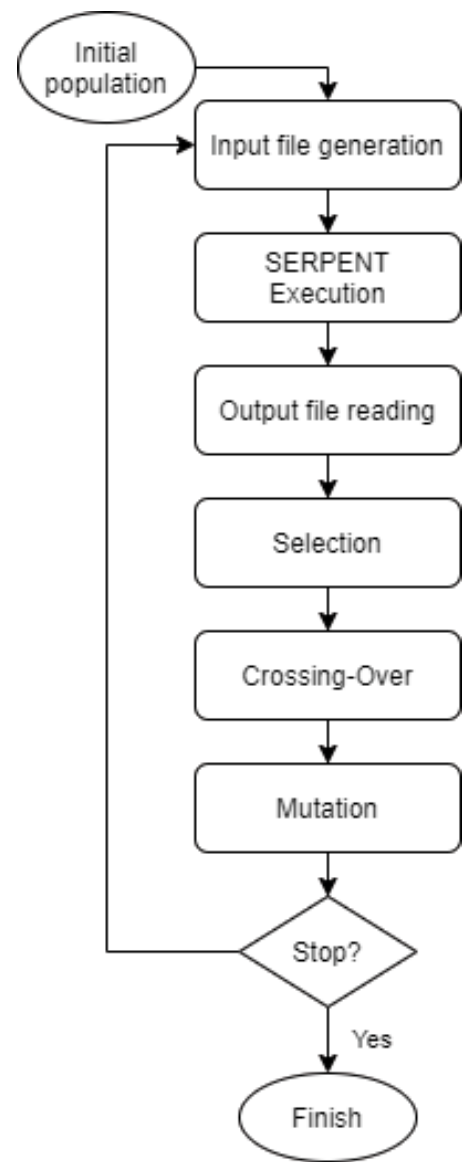

Fig. 1. General algorithm of the computational framework. the given situation, and in general, its value is higher for better individuals and lower for worse ones.

\section{Selection}

The selection operation is carried out as a draw from a collection of individuals where the probability of drawing is proportional to the FF of each individual.

\section{Crossover}

It consists of a random selection of two individuals from the population and a random selection of the place of their cut. Next, the individuals are swapped for their end parts and reunited. Supposing that $A_{1}$ and $\mathrm{A}_{2}$ are a pair of randomly chosen parents, the following equations can be arrived at:

$$
\mathrm{A}_{1}=01101 ; \mathrm{A}_{2}=11000 \text {. }
$$

Next, we need to determine the crossover point; it should be a random number between 1 and $l-1$, where $l$ is the length of the chromosome. Suppose the drawn point is 2 , then the child strings $A_{1}^{\prime}$ and $A_{2}^{\prime}$ will take the following form:

$$
A_{1}^{\prime}=01\left|000 ; A_{2}^{\prime}=11\right| 101 \text {. }
$$

Once this is done for all the parents, the individuals in the population are replaced with their children.

\section{Mutation}

This operation is realized by changing a random bit in the individual, with the probability set as a mutation rate.

\section{Implementation of GA}

The implementation of our algorithm consisted of storing numerical values of the optimized parameters as one large concatenated binary string in such a way that each individual parameter corresponded to a given subfragment of the entire string. With this solution, the algorithm at each iteration of the crossover operation exchanged substrings, which were associated with the given parameters of the models. Evaluation of a given solution consisted of reading values of coded parameters and then running simulation models with these values. The algorithm read results from output files and then used them to calculate the FF.

The end result was that those chromosomes whose binary-coded parameters generated models with better results more likely entered the next generation.

\section{Computational tool}

The fuel assembly and the reactor core models were created and then simulated with the SERPENT2 computer code, which was developed by Finnish VTT. It was applied to solve neutronics problems 
with the ENDF-VII library. It is a 3D continuous energy neutron transport Monte Carlo code with isotopic fuel depletion [19-21].

\section{Problems definition}

\section{Boron shield optimization}

In this work, we used a 3D homogeneous cylindrical core model, which was a simplified version of the SFR oxide core with 3600 MWth power and oxide fuel, defined in the Organization for Economic Cooperation and Development/Nuclear Energy Agency (OECD/NEA) benchmark [1].

For SFR reactors, it is possible that a positive void reactivity coefficient, the so-called sodium void reactivity effect (SVRE), may appear due to the removal of sodium as an effect of gas bubble entrainment or leak. This is caused by neutron spectrum hardening, which leads to an increased fission reaction rate. The SVRE can reduce core safety characteristics to the level which can potentially be dangerous during some transients. In consequence, the SVRE should be minimized during the design process [3]. The concept of utilizing boron shields in sodium reactor designs as a measure to reduce SVRE is presented in other works [22-24]. This design feature was studied in this paper. We placed boron shields around the reactor core in the form of rings partially filled with boron carbide. It is worth mentioning that boron carbide $\left(\mathrm{B}_{4} \mathrm{C}\right)$ is the most commonly used absorber in fast reactors because of its (a) relatively high neutron absorption cross sections for fast neutrons, especially with low B-10 isotope enrichment, and (b) relative low price with comparative ease of fabrication [24].

The model is schematically shown in Fig. 2 . The shade of green is related to the percentage enrichment of B-10 isotope in boron carbide, and the lighter the green colour, the greater the enrichment.

The goal was to find such a design of shields which at the same time minimizes the SVRE and does not interfere with the ability of the core to be critical. In order to achieve that, our algorithm had to manipulate selected parameters of the boron shields:

- bottom elevation

- height

- B-10 enrichment

Critical simulations were performed for two core states: normal and voided without sodium in the ac-

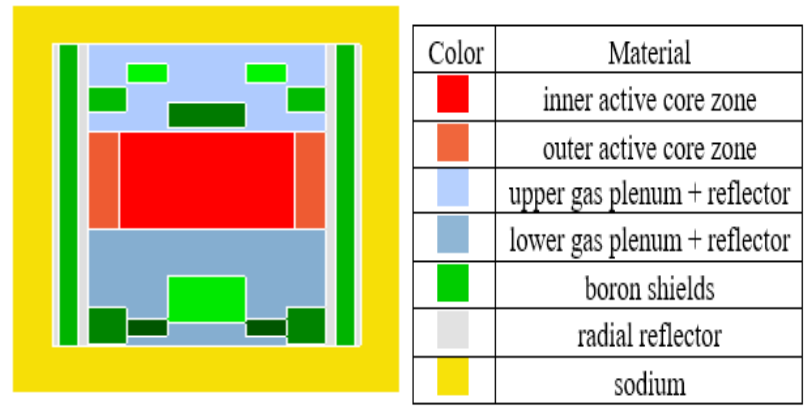

Fig. 2. Axial layout of a sample model with boron shields. tive part of the core. The resulting $k_{\text {eff }}$ values for each case will then be used to calculate sodium void reactivity (SVR), which was determined with the formula:

$$
\mathrm{SVR}=\frac{k_{\text {voided }}-k_{\text {normal }}}{k_{\text {voided }} \cdot k_{\text {normal }}} * 10^{5}
$$

where SVR is sodium void reactivity and $k$ is the effective multiplication factor. The goal is to minimize SVRE, and this means that the selected FF must increase as the SVR value decreases. The simplest example is FF $=1 / \mathrm{SVR}$. However, selecting such a FF would mean that the most preferred cores would be those that have $k_{\text {voided }}$ the same as $k_{\text {normal }}$ or very similar. As a result, the algorithm would place the thick shields as close as possible to the active part of the core, thus minimizing $k_{\text {voided }}$ but also $k_{\text {normal }}$ as well. The eigenvalue $k_{\text {normal }}<1.0$ would mean that the core is not able to maintain chain reaction. In consequence, the FF must also maximize $k_{\text {normal }}$ at the same time. The applied FF is therefore presented as follows:

$$
\mathrm{FF}=\frac{k_{\text {normal }}}{\mathrm{SVR}}-C
$$

In addition, we added constant $C$, which is given by the formula below:

$$
C= \begin{cases}0 & \text { for } n=0 \\ 0.9 * \min \left(f_{c}\right) & \text { for } n \neq 0\end{cases}
$$

where $f_{C}$ is the value of the FF of the weakest core in the initial generation. The constant $C$ was determined by an experimental method. It makes the individuals distinguishable from the roulette method so that the best ones will be selected for crossing over.

\section{Long-lived waste reduction}

The aim of this simulation was to obtain a fuel mixture with the highest possible initial $k_{\text {eff }}$ and at the same time the least amount of long-lived radioactive waste. In this task, we used a 3D model of a single fuel rod with periodic border conditions, whose geometry was derived from the OECD/NEA benchmark [1], and it is shown in Fig. 3 .

A simplified mixed oxide (MOX) fuel model was used as a basis for the mixture, in which the atomic ratio of isotopes of plutonium in the mixture was changed. During simulation, the initial content of oxygen, depleted uranium, and plutonium did not change and was found to be $2 / 3,4 / 15$, and $1 / 15$, respectively.

In the simulations, the five most stable isotopes of plutonium were taken into account, that is, those with mass numbers from 238 to 242 . The chromosome was 80 bits long, exactly 16 bits per one plutonium isotope, the value from it is read by dividing it into five equal parts, each corresponding to one of the isotopes. Then, each part is converted into an integer; finally, the isotope sum is normalized to a given value, which in this case is $1 / 15$ of the total fuel quantity. In the next step, the control program creates input files for the SERPENT code based on the values obtained in the previous step (Fig. 1).

Three actinides, americium, curium, and neptunium, were selected as the elements associated with 


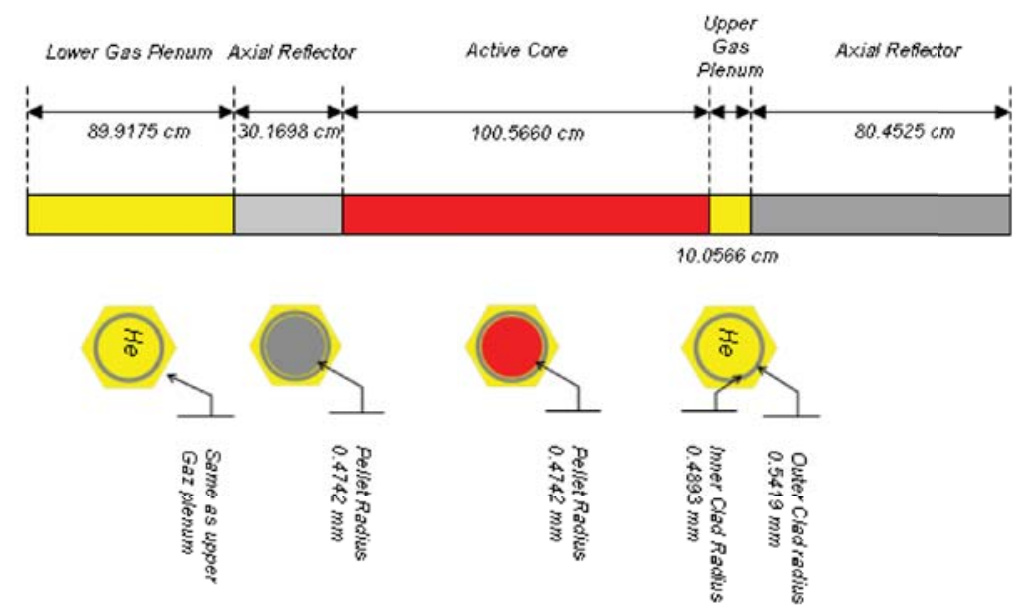

Fig. 3. Axial layout of a single fuel rod with cross sections. Taken from [1].

long-lived radioactive waste, and their concentration in spent fuel was studied. The simulation was carried out for a burnup of $100 \mathrm{MWd} / \mathrm{kgU}$, which corresponds to six years of operation in the reactor.

The developed FF is given by Eqs. (4) and (5).

$$
\begin{aligned}
& f_{C}=\left(k_{\text {eff }}, \mathrm{M}_{\text {actinides }}\right)=\frac{k_{\text {eff }}}{\mathrm{M}_{\text {actinides }}} \\
& \mathrm{M}_{\text {actinides }}=\mathrm{M}_{\mathrm{Am}}+\mathrm{M}_{\mathrm{Cm}}+\mathrm{M}_{\mathrm{Np}}
\end{aligned}
$$

Maximizing such a function will lead to maximizing the effective neutron multiplication factor and minimizing the concentration of selected actinides.

\section{Results and discussion}

\section{Boron shield optimization}

The population consisted of 20 individuals with a mutation level equal to $1 \%$, and the number of generations equal to 11 . Simulation results for the following generations are presented in Table 1. In Figs. 4 and 5, the evolution of the SVR and eigenvalues is presented. Figure 6 presents the evolution of the arrangement of the boron shields in the core.

As one can see from the results of the simulations, the algorithm has managed to obtain an optimized core in which the SVR has dropped noticeably
(Fig. 4); the SVRE was reduced from $2420 \mathrm{pcm}$ to $2130 \mathrm{pcm}$ ( $12 \%$ difference) - see Table 1 . Unfortunately, $k_{\text {normal }}$ has fallen slightly below 1 , which means that the reactor could not work in this configuration without introducing additional modifications. Apparently, the FF used in this simulation gave too little weight, maximizing the $k_{\text {normal }}$ while minimizing the SVR. The algorithm favoured individuals with the lowest possible SVR and $k_{\text {normal }}<1$ rather than individuals with lower SVR and $k_{\text {normal }}>1$ (Table 1). However, we can see that FF steadily increases; hence, the algorithms operate as designed, and the applied FF is the main limitation. In future research, the core design has to be updated with more fissile mass and updated FFs. Unfortunately, due to the limited scope of the project and necessary computing power, we could not redo simulations with more appropriate FF.

\section{Long-lived waste reduction}

The simulations were conducted with the following parameters of the GA:

- 35 Generations

- 50 Individuals per generation

- Mutation rate equals $1 \%$.

One can see in Fig. 7 that the value of the FF stabilizes around the 15th generation and remains stable until the end of the simulation, with a small

Table 1. Selected parameters of the best individual from each generation

\begin{tabular}{rcccccc}
\hline Generation & $\sum_{i} f_{1} * 10^{6}$ & $\operatorname{Max}\left(f_{i}\right)^{*} 10^{6}$ & $k_{\text {normal }}$ & $K_{\text {void }}$ & $\begin{array}{c}\text { SVR } \\
(\mathrm{pcm})\end{array}$ & $\begin{array}{c}\text { Error(SVR) } \\
(\mathrm{pcm})\end{array}$ \\
\hline 1 & 609.6 & 50.2 & 1.00376 & 1.02770 & 2422 & 12 \\
2 & 681.7 & 60.6 & 0.99744 & 1.02136 & 2348 & 12 \\
3 & 692.7 & 66.4 & 0.99703 & 1.02059 & 2316 & 12 \\
4 & 782.0 & 70.1 & 0.99526 & 1.01849 & 2292 & 12 \\
5 & 795.4 & 67.1 & 0.99798 & 1.02157 & 2314 & 12 \\
6 & 989.3 & 65.9 & 0.99654 & 1.02009 & 2317 & 12 \\
7 & 1048.3 & 66.8 & 0.99709 & 1.02063 & 2314 & 12 \\
8 & 1059.2 & 74.3 & 0.99548 & 1.01850 & 2270 & 12 \\
9 & 1088.5 & 93.7 & 0.99222 & 1.01402 & 2167 & 12 \\
10 & 1177.2 & 95.1 & 0.99391 & 1.01576 & 2164 & 12 \\
\hline
\end{tabular}




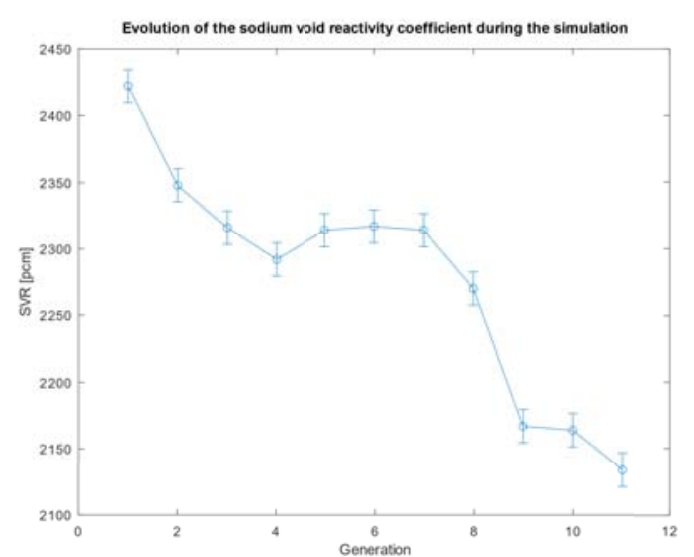

Fig. 4. SVR during the simulation.

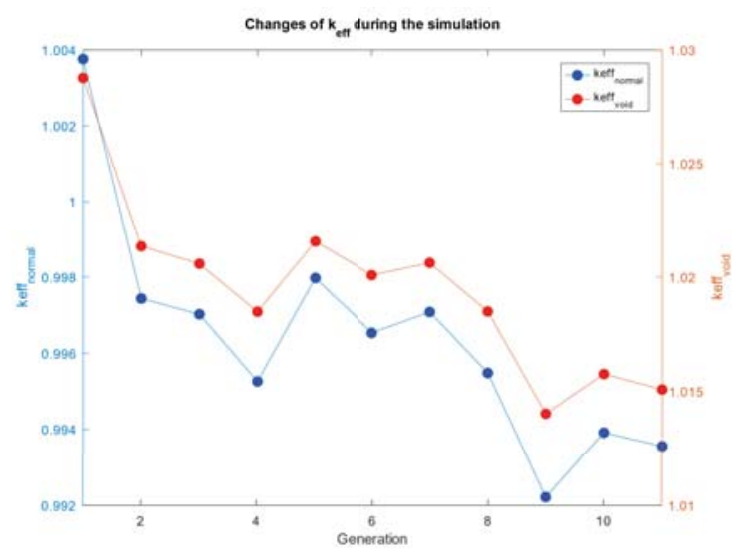

Fig. 5. Eigenvalues during the simulation.

leap in the 33rd generation. The best individual comes from the 35 th generation.

For comparison, before using the GA, simulations were performed for the mixture described in the OECD/NEA publication [1]. In consequence, the following results were obtained: the mass of selected actinides for the burnup simulation described earlier was ascertained at $\mathrm{M}_{\text {actinide }}=4.14 \mathrm{~g}$ and effective neutron multiplication factor at $k_{\text {eff }}=1.331(0.002)$. The parameters for the best individual obtained using the GA are as follows: the mass of selected actinides was ascertained at $\mathrm{M}_{\text {actinide }}=1.16 \mathrm{~g}$ and effective neutron multiplication factor at $k_{\text {eff }}=1.315(0.002)$.

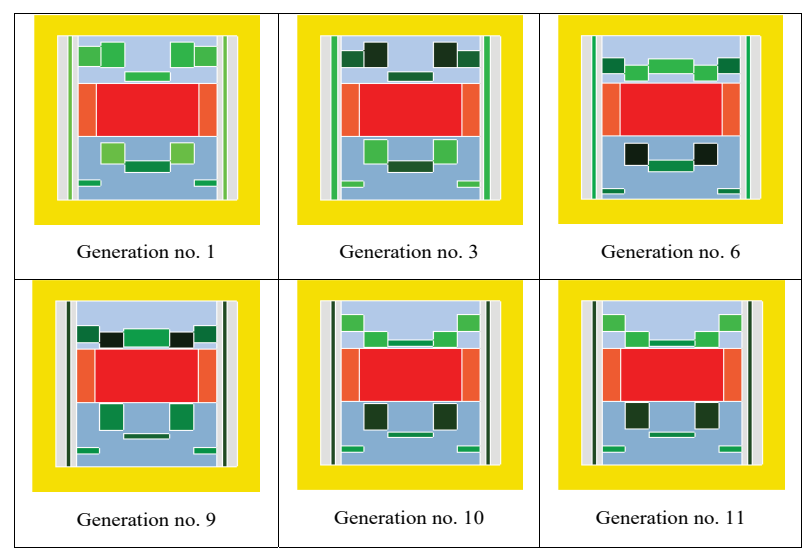

Fig. 6. Changes in the alignment of boron shields in selected generations.

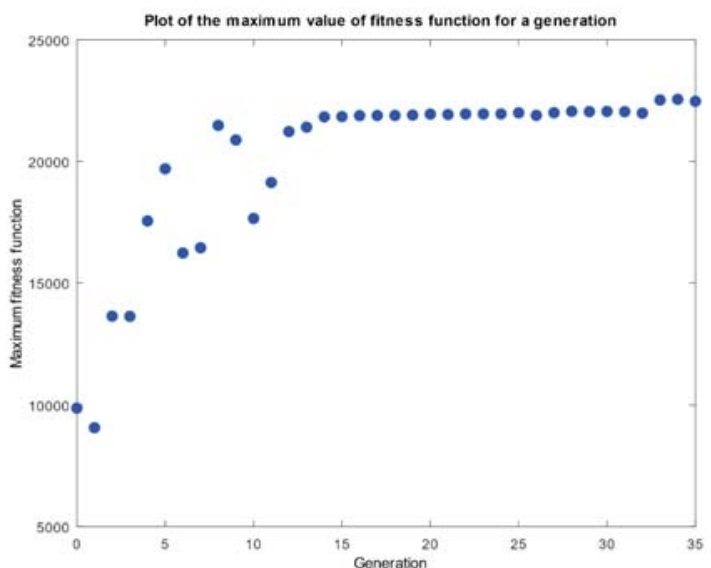

Fig. 7. Maximum fitness function value for each generation.

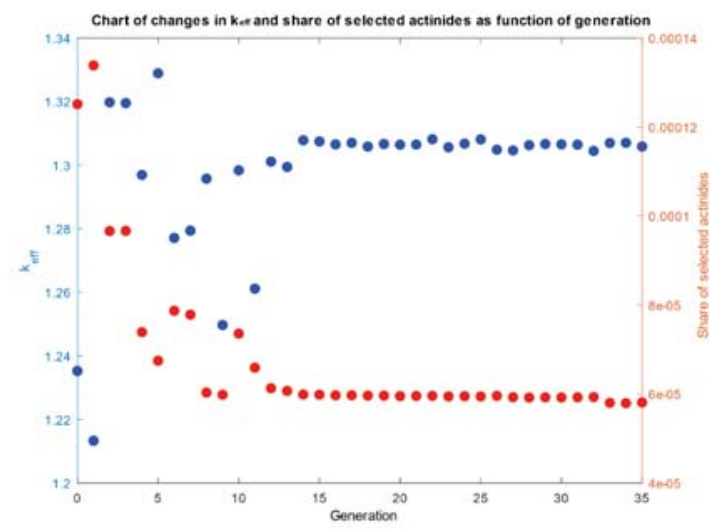

Fig. 8. The eigenvalue and concentration of selected actinides for the best individual in a generation.

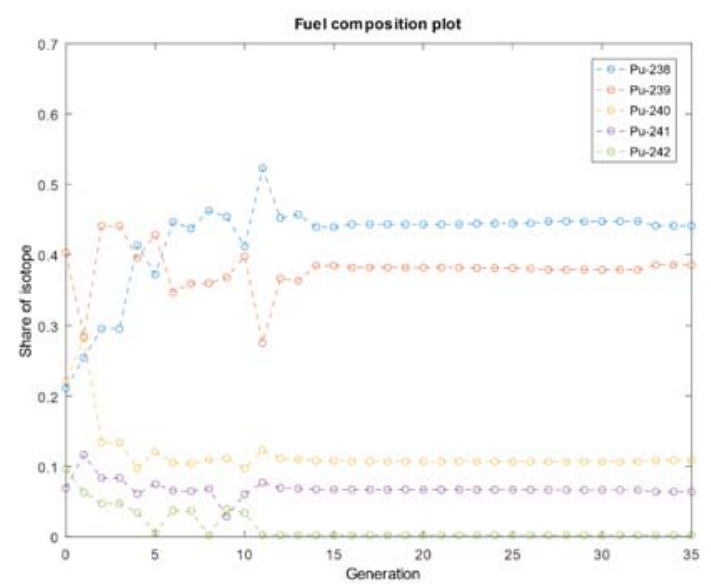

Fig. 9. Isotope composition vs. generations.

Figures 8 and 9 show the evolution of tested values for an individual with the highest FF in a given generation.

As a result of the program, a mixture of plutonium isotopes was obtained, the details of which are presented in Table 2.

The obtained mixture is dominated by the isotope of plutonium, $\mathrm{Pu}-238$, followed by $\mathrm{Pu}-239$. The algorithm resulted in the appearance of some quantities of $\mathrm{Pu}-240$ and $\mathrm{Pu}-241$ isotopes as well, and in minimization of the quantity of $\mathrm{Pu}-242$ isotope. 
Table 2. Plutonium isotope compositions

\begin{tabular}{ccccc}
\hline Pu-238 & Pu-239 & Pu-240 & Pu-241 & Pu-242 \\
\hline 0.441 & 0.385 & 0.108 & 0.064 & 0.002 \\
\hline
\end{tabular}

Table 3. Pearson's linear correlation coefficient values of plutonium isotopes

\begin{tabular}{ccccc}
\hline Pu-238 & Pu-239 & Pu-240 & Pu-241 & Pu-242 \\
\hline-0.905 & -0.090 & 0.883 & 0.629 & 0.913 \\
\hline
\end{tabular}

The obtained mixture is the result of optimization using a simple FF containing a small number of parameters. The presented solution should be treated with a proper margin of confidence. In reality, for nuclear fuel, several other factors play a role, and the obtained mixture is characterized by isotopic inventory, which is an academic case.

By collecting data from each generation, it was possible to calculate Pearson's linear correlation coefficient for the concentration of a given isotope and the content of selected actinides in the burnout fuel. The calculated values are presented in Table 3.

A negative value of the coefficient indicates a decrease in the mass of selected actinides, with an increase in the share of a given isotope. A positive value, on the other hand, indicates an increase in their mass when increasing the isotope content.

\section{Conclusions}

The SERPENT code was coupled with the GA whose purpose was to solve two nuclear engineering-related example optimization problems. Two GAs were developed and implemented in a new numerical tool. These turned out to be fully effective optimization tools, and this observation was proved by the simulations conducted. The results obtained allow us to conclude that GAs can be an efficient and easy-to-implement tool for optimizing chosen aspects of a generation IV nuclear reactor's design. However, the key to obtain the best results from GAs is to prepare a relevant FF that would reflect the environment in which the solutions are expected to be placed. Due to fact that this was only a demonstration of GAs, a set of new FFs needs to be developed if we want to use our algorithms for future research. However, nothing impedes the discussed method and tools being applied to other design problems. Practically, the only limitation in their application is the need to create appropriate FFs and computational capabilities and resources.

\section{ORCID}

P. Darnowski (10 http://orcid.org/0000-0003-1602-5379 W. Kubiński (D) http://orcid.org/0000-0002-5805-367X

\section{References}

1. NEA/OECD. (2016). Benchmark for neutronic analysis of sodium-cooled fast reactor cores with various fuel types and core sizes. OECD Nuclear Energy Agency. Available from https://www.oecdnea.org/upload/docs/application/pdf/2020-01/nscr2015-9.pdf.

2. OECD/NEA. (2014). Technology roadmap update for Generation IV nuclear energy systems. OECD Nuclear Energy Agency. Available from https://www. gen-4.org/gif/upload/docs/application/pdf/2014-03/ gif-tru2014.pdf.

3. Waltar, A. E., Todd, D. R., \& Tsvetkov, P. V. (Eds.). (2013). Fast spectrum reactors. Springer.

4. Ogawa, M. (2016). Proposals of new basic concepts on safety and radioactive waste and of new High Temperature Gas-cooled Reactor based on these basic concepts. Nucl. Eng. Des., 308, 133-141. https://doi. org/10.1016/j.nucengdes.2016.08.028.

5. El-Emam, R. S., Dincer, I., \& Zamfirescu, C. (2019). Enhanced CANDU reactor with heat upgrade for combined power and hydrogen production. Int. J. Hydrog. Energy, 44, 23580-23588. https://doi.org/10.1016/j. ijhydene.2019.06.181.

6. Yoo, J., Chang, J., Lim, J. -Y., Cheon, J. -S., Lee, T. -H., Kim, S. K., Lee, K. L., \& Joo, H. -K. (2016). Overall system description and safety characteristics of Prototype Gen IV sodium cooled fast reactor in Korea. Nucl. Eng. Technol., 48, 1059-1070. https:// doi.org/10.1016/j.net.2016.08.004.

7. Rachkov, V., \& Ashurko, Y. (2010). Review of SFR safety related operational experience. In First Joint IAEA-GIF Workshop on Operational and Safety Aspects of Sodium Cooled Fast Reactors, 23-25 May 2010, Vienna, Austria. Available from IAEA INIS database, https://inis.iaea.org/collection/NCLCollectionStore/_Public/49/104/49104075.pdf?r=1.

8. Aoto, K., Dufour, P., Hongyi, Y., Glatz, J. P., Kim, Y., Ashurko, Y., Hill, R., \& Uto, N. (2014). A summary of sodium-cooled fast reactor development. Prog. Nucl. Energy, 77, 247-265. https://doi.org/http://dx.doi. org/10.1016/j.pnucene.2014.05.008.

9. Chetal, S. C., \& Chellapandi, P. (2013). Indian fast reactor technology: Current status and future programme. Sadhana, 38(5), 795-815. https://doi. org/10.1007/s12046-013-0167-8.

10. Puthiyavinayagam, P., Selvaraj, P., Balasubramaniyan, V., Raghupathy, S., Velusamy, K., Devan, K., Nashine, B. K., Padma Kumar, G., Suresh kumar, K. V., Varatharajan, S., Mohanakrishnan, P., Srinivasan, G., \& Bhaduri, A. K. (2017). Development of fast breeder reactor technology in India. Prog. Nucl. Energy, 101, 19-42. https://doi.org/10.1016/j. pnucene.2017.03.015.

11. Holland, J. H. (1992). Adaptation in natural and artificial systems: an introductory analysis with applications to biology, control, and artificial intelligence. MIT Press.

12. Goldberg, D. E. (1979). Genetic algorithms in search, optimization, and machine learning. Addison-Wesley Publishing Company, Inc. https://www.gbv.de/dms/ ilmenau/toc/01600020X.PDF.

13. Israeli, E., \& Gilad, E. (2017). Novel genetic algorithms for loading pattern optimization using state-of-the-art operators and a simple test case. J. Nucl. Eng. Radiat. Sci., 3, 1-10. https://doi. org/10.1115/1.4035883. 
14. Hill, T. (2014). Pressurised water reactor in-core fuel management by tabu search. Ann. Nucl. Energy, 75, 64-71. https://www.repository.cam.ac.uk/bitstream/ handle/1810/245632/Tasha-Hill-revised-paper. pdf? sequence $=1$.

15. Pereira, C. M. N. A., Schirru, R., \& Martinez, A. S. (2000). Genetic algorithms applied to nuclear reactor design optimization. In Da Ruan (Ed.), Fuzzy systems and soft computing in nuclear engineering (pp. 315-334). Springer. https://doi.org/10.1007/9783-7908-1866-6_14.

16. Martín Del Campo, C., François, J. L., \& López, H. A. (2001). AXIAL: A system for boiling water reactor fuel assembly axial optimization using genetic algorithms. Ann. Nucl. Energy, 28(16), 1667-1682. https://doi.org/10.1016/S0306-4549(01)00002-0.

17. Martín-del-Campo, C., Palomera-Pérez, M. Á., \& François, J. L. (2009). Advanced and flexible genetic algorithms for BWR fuel loading pattern optimization. Ann. Nucl. Energy, 36(10), 1553-1559. https://doi. org/10.1016/j.anucene.2009.07.013.

18. Ortiz, J. J., \& Requena, I. (2004). An order coding genetic algorithm to optimize fuel reloads in a nuclear boiling water reactor. Nucl. Sci. Eng., 146(1), 88-98. https://doi.org/10.13182/NSE04-A2395.

19. Leppänen, J. (2015, June 18). Serpent - a continuousenergy Monte Carlo reactor physics burnup calcula- tion code - User's manual. Available from http:// montecarlo.vtt.fi/download/Serpent_manual.pdf.

20. Leppänen, J. (2007). Development of a new Monte Carlo reactor physics code. $\mathrm{PhD}$ Thesis, Helsinki University of Technology.

21. Leppänen, J., Pusa, M., Viitanen, T., Valtavirta, V., \& Kaltiaisenaho, T. (2015). The Serpent Monte Carlo code: Status, development and applications in 2013. Ann. Nucl. Energy, 82, 142-150. https://doi. org/10.1016/j.anucene.2014.08.024.

22. Beck, T., Blanc, V., Chapoutier, N., Escleine, J., Gauthier, L., Haubensack, D., Occhipinti, D., Pelletier, M., Phelip, M., Perrin, B., \& Venard, C. (2020). Conceptual design of fuel and radial shielding sub-assemblies for ASTRID. HAL Id: cea-02435081. Available from https://hal.archives-ouvertes.frcea-02435081.

23. Venard, C., Coquelet, C., Conti, A., Gentet, D., Lamagnere, P., Lavastre, R., Gauthe, P., Bernardin, B., Beck, T., Lorenzo, D., Scholer, Ac., \& Vernier, D. (2019). The astrid core at the end of the conceptual design phase. HAL Id: hal-02419651.

24. Guo, H., \& Buiron, L. (2018). Innovative sodium fast reactors control rod design. HAL Id: hal-01907183. Available from https://hal.archives-ouvertes.fr/hal01907183. 